\title{
Discussion on teaching of Chinese information processing
}

\author{
Chunhui Hou \\ Zhengzhou Tourism College. Zhengzhou, 450009, China \\ 34169314@qq.com
}

Keywords: Chinese, information processing; teaching; methods; strategies.

\begin{abstract}
At present, some problems of the Chinese information processing teaching are that: teachers ' teaching methods are not suitable, there are fears in students ' learning, and teaching knowledge is not standard, too random. According to the principle of unity of the students ' abilities and knowledge, the principle of teaching the dominant, student-centered principle, the principle of application-oriented, at present, Chinese information processing teaching should reform teaching knowledge, stimulate students ' enthusiasm for learning, and focus on improving and perfecting teaching methods and strategies. Therefore, this paper describes methods and strategies in the teaching of Chinese information processing.
\end{abstract}

\section{INTRODUCTION}

In the teaching of Chinese language in colleges and universities, Chinese information processing is a basic, the teaching purpose is to explore language learning based on computer-related issues and skills of formal description of language knowledge, and take advantage of natural language processing algorithms, models, basic theory of linguistic knowledge which have the skills to explore the work of the relevant industries. It will help to improve their analysis and problem-solving skills, and enhance students ' practical ability, and its role and position is critical, particularly for intelligent the computer information processing, which has critical value, its status is also crucial in the professional curriculum system that requires full attention.

\section{Comparison of teaching strategies and methods}

When teachers teach students Chinese language processing, it should be a range of algorithms and strategies, theories, and allow students to further cognitive information processing. For example, when teachers teach a disambiguation algorithm, which can require students to explain a series of algorithms, it covers maximum compression method, n-gram grammar, rules methods, cross-ambiguity ruled out, cross-ambiguity detection method, shortest path method, maximum matching method, omni word segmentation method and so on. After comparing, students can realize the advantages and disadvantages of a family of algorithms. Maximum compression method belongs to adaptive, it is marked by means of a first embodiment of training corpus, when making labels, the maximum compression ratio by way of the guide slit, a particular advantage of this method is that it adapt itself to , and the dictionary can never arise segmentation among them ; $\mathrm{n}$ primitive method through maturity n-gram statistical models and larger corpus, can improve segmentation accuracy; The method by means of appropriate rules can find the range of the entire sentence, in terms of useful information for the exclusion of very flexible, this method is more difficult to obtain knowledge , if only by virtue of the rules were written, which inevitably increases the workload, and it is difficult to conduct a comprehensive summary ; cross ambiguity exclusion method based on the memories of strategy, pseudo ambiguity word segmentation field table shows, can greatly improve the crossing ambiguity segmentation accuracy, moreover, for the part of the bias severe real ambiguity field, can further improve the accuracy of crossing ambiguity segmentation; Cross ambiguity detection will detect the method, all cross ambiguity result of sentences are given, which can effectively improve efficiency. The shortest path method of dynamic programming methods by word graph among the starting point to the end to find the shortest path, with maximum match method compared, the method 
can achieve the desired effect, however, which is also facing the omission of the phenomenon; maximum matching method is difficult to be covered by ambiguity, which may be missing some of the complexity of the cross ambiguity; whole segmentation algorithm can be given a sentence segmentation result of anything that would appear, however, this method requires a large overhead.

\section{Interactive teaching strategies and methods}

Interactive teaching refers to the teaching practice as the implementation of a process of communication and interaction, the whole process of teaching as a dynamic combination of interactive teaching activities and interaction , under the mutual influence of teachers and students , harmony the formation of teacher-student interaction, thus improving the quality of teaching efficiency and a teaching method .

Stark, who presented by "listening" to learn, some time to learn, and learn through their own feelings are basically learning.It covers the significance of the two aspects, one that only a professor teaching the teachers, the students listened to one, which is very one-sided, and it is difficult to determine how much students to learn and how much teachers to teach, but only in some cases, it is true to learn; On the other hand, has a very large number of alternative teacher professor teaching model, which requires students to experience the process of solving the problem, and thus explore the answer to the question, which is very effective, because the method allows the student to study. A lot of information research data reflected, when teachers teach the students ' knowledge, fewer students had been able to acquire knowledge, but students through its own process of thinking and inquiry results, coupled with the teacher's instruction, students will be more impressed with the learning, students ' perceptions are very system, and it also helps the formation of good study habits.

When teachers teach the students ' knowledge of Chinese information processing, based on interactive teaching, both in test areas, and professor in the theory of knowledge, according to the steps are shown in Figure 1,

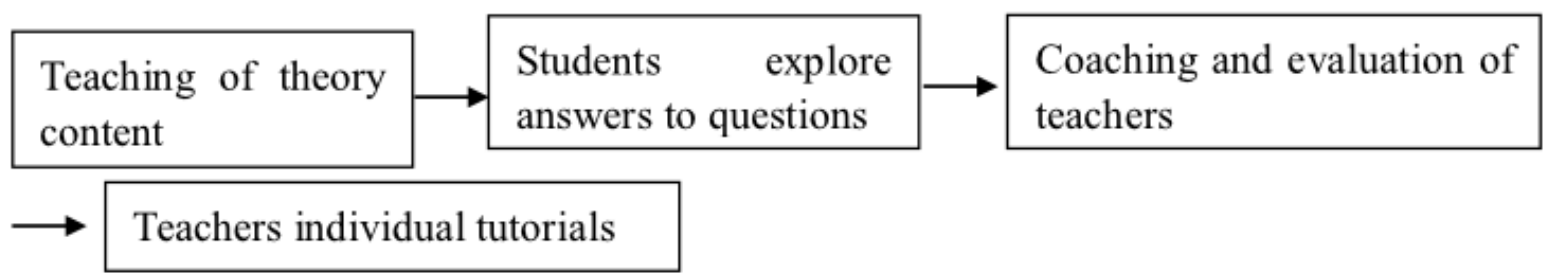

Figure 1 (specific teaching steps of interactive teaching)

Among them, the teachers teach students theory, which is the basis for ensuring interaction, and teachers give students a test or assignment, this step is critical, whether the teacher can select appropriate test and thinking questions is the key, and teachers effective coaching and evaluation is an important factor in ensuring the interaction of teachers. After that, individual counseling is to safeguard and consolidate the interaction outcomes.

Interactive teaching model can help students have more to do with physical access and participation, in participating in the process of research results or answers to your questions. Facts show that the teachers implement interactive teaching, learning and teaching to help students gain a deeper knowledge, which can also learn more about the technology and research progress of this course, and enjoy their theoretical content.

\section{Classified teaching strategies and methods}

Some is an extension of nature, its role is to let students more deep to extracurricular reference literature for reading; Some are inspired by nature, its role is to arouse students' independent thinking, so that law students to explore language skills improve; Some belong to consolidate nature, its role is to consolidate the learned content; some belong to the integrated nature of its role is to enable 
students to mastery of the learning content. After conducting classification, learning becomes more convenient, which will also help students to summarize and improve induction capabilities.

Because it is based on the analysis and research of computer technology to Chinese information processing, which is different from the language word processing and research carried out by students, and Chinese information processing, information processing, text processing, sentence words, Chinese character information processing has been involved in all aspects of information processing, which requires students to categorize knowledge so that students learn to pierce previous knowledge. Because of changing the object of research-oriented, parsing of language processing was a very severe challenges: the traditional sense of grammatical analysis for the people belong to the description on the experience, explaining the type, while computational linguistics, machine-oriented operation experiment technology type of analysis, which requires parsing rules to be formalized in the traditional sense, demonstrated by being able to calculate means. Because changing the aim of analysis, syntax analysis needs of information processing theory based on rules and scope both for more formal content. Because conversion analysis, so it has greatly accelerated the pace of analysis of modern Chinese grammar and also to come up with a more comprehensive, systematic and scientific rules and categories. Because conversion of analysis point of view, we can discover the many unanswered questions, as well as investigate the problems towards inherent problems. In summary, the results obtained for the analysis of human language is related to human natural language content, usually expressed through natural language, machine-oriented language analysis focuses on the implementation of a formal inquiry under the framework, the content reflection of the analysis language is formalized. Among them, the human-oriented nature of linguistic analysis is mostly illustrations, and machine-oriented language analysis with particular emphasis on coverage of corpus and language knowledge system, which language shall implement the appropriate size. Table 1 shows the traditional sense of grammatical analysis of human-oriented analysis and computational linguistics machine-oriented similarities and differences:

Table 1 (as shown in the traditional sense of grammatical analysis and computer linguistics for machine-oriented analysis of the similarities and differences of people)

\begin{tabular}{|c|c|c|}
\hline area & $\begin{array}{c}\text { analysis of traditional grammar-oriented } \\
\text { people }\end{array}$ & $\begin{array}{c}\text { computational linguistics analysis of } \\
\text { machine-oriented }\end{array}$ \\
\hline type & $\begin{array}{c}\text { description of experience, explanation of } \\
\text { the type }\end{array}$ & $\begin{array}{c}\text { type of operation experimental } \\
\text { technology }\end{array}$ \\
\hline expression & natural language & formal \\
\hline nature & illustration nature & $\begin{array}{c}\text { coverage of corpus and systematic of } \\
\text { knowledge }\end{array}$ \\
\hline
\end{tabular}

\section{Conclusion}

The teaching practice shows that the Chinese information processing is very effective, and we will work harder, and constantly update teaching ideas in teaching practice, which continues to explore new strategies and methods of teaching Chinese information processing.

\section{References}

[1] Zhang Wen. Analysis the meaning of the Corpus building for language teaching in teaching Chinese as a foreign language, for example. [J] Northern Literature ( second half ) .2011 ( 11 ) .

[2] Zhang Ruixia, Zhuang Jinlin, Yang Guozeng. based on hownet disambiguation study of Chinese information structure [j]. Journal of Chinese information. 2012 (04) 